\title{
Effects of low glycemic index/high-fat, high-calorie diet compared to the high-fat, high-calorie diet on glycemic control, lipid profile, and inflammatory markers in children and adolescence with cystic fibrosis: A study protocol for a randomized double- blind controlled clinical trial
}

\section{Zahra Gorji}

Tehran University of Medical Sciences

Mohammadreza Modaresi

Tehran University of Medical Sciences

Saeed Yekanni-Nejad

Tehran University of Medical Sciences

Maryam Mahmoudi ( $\square$ mahmoodi_maryam@yahoo.com )

Study protocol

Keywords: Cystic fibrosis, Glycemic index, Glycemic control, Inflammation, Lipid profile

Posted Date: October 30th, 2019

DOI: https://doi.org/10.21203/rs.2.16168/v1

License: (c) (1) This work is licensed under a Creative Commons Attribution 4.0 International License.

Read Full License 


\section{Abstract}

Background: Inflammation plays an important role in pathogenesis of cystic fibrosis, and diet is considered as an effective factor in controlling inflammation. Low glycemic index diet seems to be effective for improving glycemic control and reducing the inflammation and lipid profile. Hence, this study was planned to compare the effects of a low glycemic index/high fat, high calorie diet with the high fat, high calorie diet on glycemic status, lipid profile, and inflammatory markers in patients with cystic fibrosis.

Methods: A total of 60 children and adolescence with cystic fibrosis will be randomized to receive a high fat, high calorie diet $(n=30)$ or low glycemic index/high fat, high calorie diet $(n=30)$ with similar calorie and macronutrients composition, for three months. Patients in high fat, high calorie diet arm will be able to use all sources of carbohydrates with different glycaemic indices; while those in another arm will be received their carbohydrate from low glycemic index sources. Before and after the intervention, serum levels of lipid profile (triglyceride, total cholesterol, HDL cholesterol, LDL cholesterol), insulin, glucose, $\mathrm{HbA1c}$, the homeostasis model assessment-estimated insulin resistance index, and some inflammatory markers (IL-6, IL-10, IL-17A, and IFNY) will be measured.

Discussion: To our knowledge, this study will be the first to examine the effects of a low glycemic index/high fat, high calorie diet compared to the high fat, high calorie diet in cystic fibrosis patients. The findings of this study might provide evidence to improve the nutritional status and immune system as well as to prevent the disease complications in these patients.

\section{Background}

Cystic fibrosis (CF) is an autosomal recessive inherited disorder which caused by a defect in the CF transmembrane conductance regulator (CFTR) on chromosome 7 [1]. This autosomal genetic disease occurs in a raising trend worldwide and it is incidence is reported to be approximately 1 in 3000 births [2, 3].

Individuals with CF develop a wide spectrum of complications, of which, exocrine pancreatic dysfunction is one of the most common manifestations, found in $85 \%$ of patients [4]. This type of dysfunction leads to fat malabsorption and in turn results in fat-soluble vitamin inadequacy, malnutrition, and growth failure $[5,6]$. CF-related diabetes (CFRD) is another hallmark of CF manifestations which directly linked to increased morbidity and mortality of CF [7]. This complication has been shown to be related to a decline in lung function [8] and also increased risk of bacterial chest infections [9]. Moreover, patients with both CF and CFRD have higher levels of inflammatory markers, and it has been shown that inflammatory biomarkers might be associated with complications of both CF and CFRD [10-13]. Hence, the strategies to reduce inflammatory markers are expected to be beneficial in prevention of CF-related complications [14]. 
Diet is considered as an effective factor in controlling inflammation. It has been shown that high glycemic index (GI) diet increase oxidative stress and inflammation [15-17], while low GI diet has been indicated potential for improving glycemic control and lipid profile in other forms of diabetes [18-21]. Also, previous studies have shown a beneficial effect of low GI diet on glycemic control and lipid profile in individuals with different health conditions $[22,23]$. However, the evidence for the use of low GI diet in CFRD is scare. Given that hyperglycemia exacerbates the clinical situation in CF patients by induction of immune responses, it seems that low GI diet can be an effective factor in modulating the immune response in CF patients by controlling glucose homeostasis [14]. Moreover, given that $16 \%$ of CF children may suffer from diabetes and hyperlipidemia, administration of low GI diet might be benefit for these children [24].

As the primary goal of nutrition therapy in $\mathrm{CF}$ is to achieve optimal weight gain and growth, a high fat and high calorie diet has been recommended for these patients [25]. Regarding a definite necessity for determination of the standard diet with the optimal effect on growth, survival rate, quality of life, and the reduction of complications in CF patients, we designed a study to compare the effects of low $\mathrm{GI} / \mathrm{high}$ fat, high calorie diet with the high fat, high calorie diet on glycemic status, lipid profile, and inflammation markers in patients with CF. We hypothesized that low GI diet will improve the glycemic status and reduce the inflammation and lipid profile in children and adolescence with CF.

\section{Methods/design \\ Study design}

\section{This is a randomized, double-blinded, parallel group, clinical trial. The study protocol was registered in the Iranian Registry of Clinical Trial (registration No, IRCT2017102325267N5).}

\section{Study population}

This study will be conducted on children and adolescents with CF who are referred to the Cystic Fibrosis Clinic of the Children's Medical Center, affiliated to Tehran University of Medical Sciences, Tehran, Iran. All children and adolescents, aged 6-18 years old, who are diagnosed with CF by a sweat test or genetic test by a clinical specialist, will be included. Also, we will check oral glucose tolerance test (OGTT) levels from participants' medical records and those with normal OGTT levels and with no CFRD will be included in the study. The participants will be excluded if: 1) have other chronic diseases such as diabetes and thyroid disease, liver diseases (i.e., hepatitis and cirrhosis), 2) were hospitalized at the beginning of the study or one month earlier, 3) had a history of surgery one month prior to the intervention or were supposed to undergo surgery (except for dental surgery) in the next three months, and 4) take steroids. Moreover, the 
participants will be excluded if they will not willing to follow the prescribed diets, unable to adhere to the diets, hospitalize or require surgery during the study.

\section{Sample size}

Sample size was determined by formula suggested for randomized clinical trials, with type I error of $5 \%$, type II error of $20 \%$, study power of $80 \%$, and serum interleukin-17A (IL-17A) level as a key variable [26]. The number of needed samples was calculated as 21 participants. To get a more confident result with a $30 \%$ dropout rate, we will consider 30 participants in each group.

\section{Randomization}

All eligible participants will be enrolled by convenience sampling method and will be randomly (1:1 ratio) allocated by an assistant to high fat, high-calorie diet (HFHC; $n=30$ ) or low $\mathrm{Gl} /$ high fat, high calorie diet $(L G ; n=30)$, using permuted block randomization method, stratified by age and sex.

\section{Blinding}

Given the nature of the intervention (diet), investigators will not be blinded to the intervention. However, all outcomes will be measured by an independent assessor who will be blind to the group allocation. Also, patients and data analysts will be blinded to the group allocation.

\section{Procedure and intervention}

In both group, the intervention will be done during three months and both groups will be visited at baseline and after three months of study. All patients will be received a leaflet, presenting the number of portions per meal, food exchange list, food recommendations, and allowed and forbidden foods (for LG diet only).

To design a diet for CF children and adolescents, we first calculate the required energy intake of participants according to the Paediatrics Clinics formula [27]. Then, we added further 300 to calculated energy. The patients in the HFHC diet arm will be received a high-fat, high-calorie diet containing $40 \%$ fat, $20 \%$ protein, and $40 \%$ carbohydrate. This group will be able to use all sources of carbohydrates with different Gls. The patients in the LG diet arm will be received a high-fat, high-calorie diet with macronutrients percentage similar to that in HFHC group; however, the difference will be that the carbohydrate sources were supplied from low GI sources. LG and high glycemic index (HGI) were defined as $\mathrm{GI}<50$ and $\mathrm{GI} \geq 50$, respectively. The low $\mathrm{Gl}$ diet group will be instructed to select carbohydrate containing foods from a list of low GI grains, fruits, vegetables, and dairy, which was provided by the study researchers. Moreover, a list of prohibited HGI foods will be given to members of this group. 
The criterion for compliance in the low GI group will be $\mathrm{Gl}<50$, calculated from the food diaries. To extract GI values, we will refer to the Iranian GI table [28], and the international table of GI [29] will be used for GI values not reported on the Iranian-specific table. The GI for foods not included in the Iranian or the international table will estimate using the GI for the most similar food. To calculate the mean GI for diet, we will use the reported formula [30].

\section{Measurement}

\section{General information}

Information regarding socio-demographic background, current use of medications and supplements, other medical interventions related to the treatment, and lack of allergy or intolerance to certain foods will be recorded by participants' medical record and also interviews at baseline.

\section{Outcomes}

The primary outcomes are changes in the following parameters at the end of the study in comparison with the baseline values: Hemoglobin A1c (HbA1c); fasting blood glucose (FBS); insulin; homeostasis model assessment-estimated insulin resistance (HOMA-IR) index; lipid profile such as triglyceride (TG), total cholesterol (TC), high-density lipoprotein (HDL) cholesterol, low-density lipoprotein (LDL) cholesterol; and inflammatory markers such as interleukin 6 (IL-6), interleukin $10(\mathrm{IL}-10), \mathrm{IL}-17 \mathrm{~A}$, and interferongamma (IFNY).

The primary variables will be measure at the beginning and the end of the study. For this purpose, venous blood sample $(10 \mathrm{~mL})$ will be drawn after 12-h overnight fasting by trained nurses in seated position to measure biochemical markers. Then, blood samples will be centrifuged at $3000 \mathrm{rpm}$ for $10 \mathrm{~min}$ at $4{ }^{\circ} \mathrm{C}$ to obtain serum which will be stored at $-80^{\circ} \mathrm{C}$ until biochemical analyses. Lipid profiles and serum levels of glucose will be measured by the enzymatic colorimetric method using standard kits.

HbA1c concentrations will be measured by using an auto-analyzer (Selectra E, Vitalab, Holliston, the Netherlands). Serum insulin will be measured using enzyme-linked immunosorbent assay (ELISA) kit. Insulin resistance will be determined using the HOMA-IR equation. Serum levels of IL-6, IL-10, IL-17A, and IFNY will be measured using ELISA kits.

\section{Secondary outcomes}

The secondary outcomes are changes in weight and body mass index (BMI) which will be measure at the beginning and the end of the study. Height will be measured using the SECA stadiometer to the nearest $0.1 \mathrm{~cm}$ and weight will be measured using a SECA electronic scale to the nearest $0.1 \mathrm{~kg}$. BMI will be calculated as weight $(\mathrm{kg})$ divided by height squared $\left(\mathrm{m}^{2}\right)$. 


\section{Adherence}

All patients will be trained to follow the instructions of the diets. At the beginning and the end of the study, dietary information of all participants will be collected using a 24-hour dietary recall in three nonconsecutive days, and will be analysed by Nutritionist IV software (First Databank, San Bruno, CA). To assess participants adherence during study period, a dietitian will be assigned to check them weekly using telephone. Patients will be monitored weekly during the study period and any occurrence of adverse events will be recorded.

\section{Statistical analysis}

Continues and categorical variables will be reported as mean \pm standard deviation and number and percentages, respectively. Kolmogorov-Smirnov test will be applied to explore the normality distribution of variables. Paired sample t-test will be performed for within-group changes comparisons (baseline vs. post-intervention values). The analysis of covariance (ANCOVA), will be adjusted for baseline values and will be used for evaluation of between-group differences. All outcome variables will be assessed based on intention-to-treat and per protocol analysis. Missing data will be entered using "regression imputation" method. The level of significance will set at a probability of $<0.05$ for all analyses. All data analyses will be conducted using the statistical package for social sciences, version 23.0 (SPSS, Chicago, IL, U.S.A).

\section{Discussion}

$\mathrm{CF}$ is an autosomal recessive inherited disorder which affects many organs, including the lungs, the digestive tract, and various epithelial tissues [31]. Generally, CF is an inflammatory disorder and since inflammation is associated with an increased risk of mortality, the strategies to reduce inflammatory markers is expected to be beneficial in prevention of this chronic disease [14].

Diet is an effective factor in controlling inflammation in many diseases. Many interventional and observational studies have emphasized the effects of different types of carbohydrate on acute and chronic inflammation $[15,17]$. It has been shown that high $\mathrm{Gl}$ foods increase oxidative stress and inflammation [15-17], while low GI diets improve glycemic control and lipid profile [18-21]. However, the evidence for the use of low GI diet in CF is scare. According to our knowledge, there is no previous study that compared the effects of low $\mathrm{Gl} /$ high fat, high calorie diet compared to the high fat, high calorie diet in patients with CF. The findings of this study might provide evidence to improve the nutritional status and immune system as well as to prevent the disease complications in these patients.

\section{Trail status}

Authors confirmed that the recruitment has been begun in $15^{\text {th }}$ Dec, 2018 and expected that recruitment will be completed in $20^{\text {th }} \mathrm{Dec}, 2019$. The flow chart of the study protocol and the time schedule of 
enrolment, interventions, assessments, and visits for participants are indicated in Figures 1 and 2.

\section{Abbreviations}

ANCOVA: Analysis of covariance; BMI: Body mass index; CF: Cystic fibrosis; CFRD: Cystic fibrosis-related diabetes mellitus; CFTR: CF transmembrane conductance regulator; ELISA: Enzyme-linked immunosorbent assay; GI: Glycemic index; FBG: Fasting blood glucose; HbA1c: Hemoglobin A1c; HDL: High-density lipoprotein; HFHC: High-fat, high-calorie diet; HGl: High glycemic index; HOMA-IR: Homeostasis model assessment-estimated insulin resistance index; IFNY: interferon-gamma; IL: Interleukin; LDL: Low-density lipoprotein; LG: Low-glycemic index/high-fat, high-calorie diet; OGTT: Oral glucose tolerance test; TG: Triglyceride; TC: Total cholesterol.

\section{Declarations}

\section{Acknowledgments}

\section{The authors thank all mangers and staff of the Cystic Fibrosis Clinic of the Children's Medical Center.}

\section{Authors' contributions}

ZG designed the initial idea of this work, which was further developed by MM and MM. SY advised on statistical analysis. ZG organized participant management and data collection. ZG and MM drafted the manuscript. The manuscript has been read and approved by all authors.

\section{Funding}

This study has been supported by Tehran University of Medical Sciences and Health Services (Project No. 9423324002). The funder has no role in study design, collection, analysis, and interpretation of data, writing of the paper, or the decision to submit the paper.

\section{Availability of data and materials}

\section{Not applicable.}

\section{Ethics approval and consent to participate}


The local Ethics Committee of Tehran University of Medical Sciences has approved study protocol (IR.TUMS.VCR.REC.1396.3171). A written informed consent form will be obtained before participating in the study by investigator. Whenever a person is unable to continue supplementation, he/she will be excluded from the study.

\section{Consent for publication}

\section{Not applicable.}

\section{Competing interests}

\section{The authors declare that they have no competing interests.}

\section{References}

1.Chiu WT, Tran TV, Pan SC, Huang HK, Chen YC, Wong TW. Cystic Fibrosis Transmembrane Conductance Regulator: A Possible New Target for Photodynamic Therapy Enhances Wound Healing. Adv Wound Care (New Rochelle). 2019;8(10):476-86. Doi: 10.1089/wound.2018.0927.

2.Parker-McGill K, Nugent M, Bersie R, Hoffman G, Rock M, Baker M, et al. Changing incidence of cystic fibrosis in Wisconsin, USA. Pediatr Pulmonol. 2015;50(11):1065-72. Doi: 10.1002/ppul.23265.

3.Hangul M, Pekcan S, Kose M, Acican D, Sahlar TE, Erdogan M, et al. The Incidence of Cystic Fibrosis in the Central Region of Anatolia in Turkey Between 2015 and 2016. Balkan Med J. 2019;36(3):179-83. Doi: 10.4274/balkanmedj.galenos.2018.2018.1332.

4.Papas KA, Sontag MK, Pardee C, Sokol RJ, Sagel SD, Accurso FJ, et al. A pilot study on the safety and efficacy of a novel antioxidant rich formulation in patients with cystic fibrosis. J Cyst Fibros. 2008;7(1):60-7. Doi: 10.1016/j.jcf.2007.05.001.

5.Kleme ML, Levy E. Cystic fibrosis-related oxidative stress and intestinal lipid disorders. Antioxid Redox Signal. 2015;22(7):614-31. Doi: 10.1089/ars.2014.6012.

6.Kalivianakis M, Minich DM, Bijleveld CM, van Aalderen WM, Stellaard F, Laseur M, et al. Fat malabsorption in cystic fibrosis patients receiving enzyme replacement therapy is due to impaired intestinal uptake of long-chain fatty acids. Am J Clin Nutr. 1999;69(1):127-34. Doi:

10.1093/ajcn/69.1.127.

7.Perrem L, Stanojevic S, Solomon M, Carpenter S, Ratjen F. Incidence and risk factors of paediatric cystic fibrosis-related diabetes. J Cyst Fibros. 2019. Doi: 10.1016/j.jcf.2019.04.015. 
8.Milla CE, Warwick WJ, Moran A. Trends in pulmonary function in patients with cystic fibrosis correlate with the degree of glucose intolerance at baseline. Am J Respir Crit Care Med. 2000;162(3 Pt 1):891-5. Doi: $10.1164 /$ ajrccm.162.3.9904075.

9.Brennan AL, Gyi KM, Wood DM, Johnson J, Holliman R, Baines DL, et al. Airway glucose concentrations and effect on growth of respiratory pathogens in cystic fibrosis. J Cyst Fibros. 2007;6(2):101-9. Doi: 10.1016/j.jcf.2006.03.009.

10.Romeo GR, Lee J, Shoelson SE. Metabolic syndrome, insulin resistance, and roles of inflammationmechanisms and therapeutic targets. Arterioscler Thromb Vasc Biol. 2012;32(8):1771-6. Doi: 10.1161/atvbaha.111.241869.

11.Nichols DP, Chmiel JF. Inflammation and its genesis in cystic fibrosis. Pediatr Pulmonol. 2015;50 Suppl 40:S39-56. Doi: 10.1002/ppul.23242.

12.Quinton PM. Cystic fibrosis: lessons from the sweat gland. Physiology (Bethesda). 2007;22:212-25. Doi: 10.1152/physiol.00041.2006.

13.Cantin AM, Hartl D, Konstan MW, Chmiel JF. Inflammation in cystic fibrosis lung disease: Pathogenesis and therapy. J Cyst Fibros. 2015;14(4):419-30. Doi: 10.1016/j.jcf.2015.03.003.

14.Ziai S, Coriati A, Gauthier MS, Rabasa-Lhoret R, Richter MV. Could T cells be involved in lung deterioration and hyperglycemia in cystic fibrosis? Diabetes Res Clin Pract. 2014;105(1):22-9. Doi: 10.1016/j.diabres.2014.03.002.

15.Buyken AE, Goletzke J, Joslowski G, Felbick A, Cheng G, Herder C, et al. Association between carbohydrate quality and inflammatory markers: systematic review of observational and interventional studies. Am J Clin Nutr. 2014;99(4):813-33. Doi: 10.3945/ajcn.113.074252.

16. Hu Y, Block G, Norkus EP, Morrow JD, Dietrich M, Hudes M. Relations of glycemic index and glycemic load with plasma oxidative stress markers. Am J Clin Nutr. 2006;84(1):70-6; quiz 266-7. Doi: 10.1093/ajcn/84.1.70.

17.Rouhani MH, Kelishadi R, Hashemipour M, Esmaillzadeh A, Surkan PJ, Keshavarz A, et al. The Impact of a Low Glycemic Index Diet on Inflammatory Markers and Serum Adiponectin Concentration in Adolescent Overweight and Obese Girls: A Randomized Clinical Trial. Horm Metab Res. 2016;48(4):2516. Doi: $10.1055 / \mathrm{s}-0042-100467$.

18.Esfahani A, Wong JM, Mirrahimi A, Srichaikul K, Jenkins DJ, Kendall CW. The glycemic index: physiological significance. J Am Coll Nutr. 2009;28 Suppl:439s-45s. Doi:

19.Gilbertson HR, Brand-Miller JC, Thorburn AW, Evans S, Chondros P, Werther GA. The effect of flexible low glycemic index dietary advice versus measured carbohydrate exchange diets on glycemic control in children with type 1 diabetes. Diabetes Care. 2001;24(7):1137-43. Doi: 10.2337/diacare.24.7.1137. 
20.Jarvi AE, Karlstrom BE, Granfeldt YE, Bjorck IE, Asp NG, Vessby BO. Improved glycemic control and lipid profile and normalized fibrinolytic activity on a low-glycemic index diet in type 2 diabetic patients. Diabetes Care. 1999;22(1):10-8. Doi: 10.2337/diacare.22.1.10.

21.Jenkins DJ, Kendall CW, McKeown-Eyssen G, Josse RG, Silverberg J, Booth GL, et al. Effect of a lowglycemic index or a high-cereal fiber diet on type 2 diabetes: a randomized trial. Jama.

2008;300(23):2742-53. Doi: 10.1001/jama.2008.808.

22.Markovic TP, Muirhead R, Overs S, Ross GP, Louie JC, Kizirian N, et al. Randomized Controlled Trial Investigating the Effects of a Low-Glycemic Index Diet on Pregnancy Outcomes in Women at High Risk of Gestational Diabetes Mellitus: The GI Baby 3 Study. Diabetes Care. 2016;39(1):31-8. Doi: 10.2337/dc150572 .

23.0jo 0, Ojo 00, Wang XH, Adegboye ARA. The Effects of a Low GI Diet on Cardiometabolic and Inflammatory Parameters in Patients with Type 2 and Gestational Diabetes: A Systematic Review and Meta-Analysis of Randomised Controlled Trials. Nutrients. 2019;11(7). Doi: 10.3390/nu11071584.

24.Juanola-Falgarona M, Salas-Salvado J, Ibarrola-Jurado N, Rabassa-Soler A, Diaz-Lopez A, GuaschFerre $\mathrm{M}$, et al. Effect of the glycemic index of the diet on weight loss, modulation of satiety, inflammation, and other metabolic risk factors: a randomized controlled trial. Am J Clin Nutr. 2014;100(1):27-35. Doi: 10.3945/ajcn.113.081216.

25.Lai HC, Kosorok MR, Laxova A, Davis LA, FitzSimmon SC, Farrell PM. Nutritional status of patients with cystic fibrosis with meconium ileus: a comparison with patients without meconium ileus and diagnosed early through neonatal screening. Pediatrics. 2000;105(1 Pt 1):53-61. Doi:

10.1542/peds.105.1.53.

26.Karabulut S, Afsar CU, Karabulut M, Alis H, Kilic L, Cikot M, et al. Evaluation of Serum Interleukin-17 (IL-17) Levels as a Diagnostic Marker in Pancreatic Adenocarcinoma. J Gastrointest Cancer. 2016;47(1):47-54. Doi: 10.1007/s12029-015-9787-z.

27.Lusman S, Sullivan J. Nutrition and Growth in Cystic Fibrosis. Pediatr Clin North Am. 2016;63(4):66178. Doi: 10.1016/j.pcl.2016.04.005.

28.Azadbakht L, Mohammadifard N, Akhavanzanjani M, Taheri M, Golshahi J, Haghighatdoost F. The association between dietary glycemic index, glycemic load and diet quality indices in Iranian adults: results from Isfahan Healthy Heart Program. Int J Food Sci Nutr. 2016;67(2):161-9. Doi: 10.3109/09637486.2015.1134443.

29.Foster-Powell K, Holt SH, Brand-Miller JC. International table of glycemic index and glycemic load values: 2002. Am J Clin Nutr. 2002;76(1):5-56. Doi: 10.1093/ajcn/76.1.5. 
30.De Rougemont A, Normand S, Nazare JA, Skilton MR, Sothier M, Vinoy S, et al. Beneficial effects of a 5-week low-glycaemic index regimen on weight control and cardiovascular risk factors in overweight nondiabetic subjects. Br J Nutr. 2007;98(6):1288-98. Doi: 10.1017/s0007114507778674.

31.Riordan JR, Rommens JM, Kerem B, Alon N, Rozmahel R, Grzelczak Z, et al. Identification of the cystic fibrosis gene: cloning and characterization of complementary DNA. Science. 1989;245(4922):1066-73. Doi: 10.1126/science.2475911.

\section{Figures}

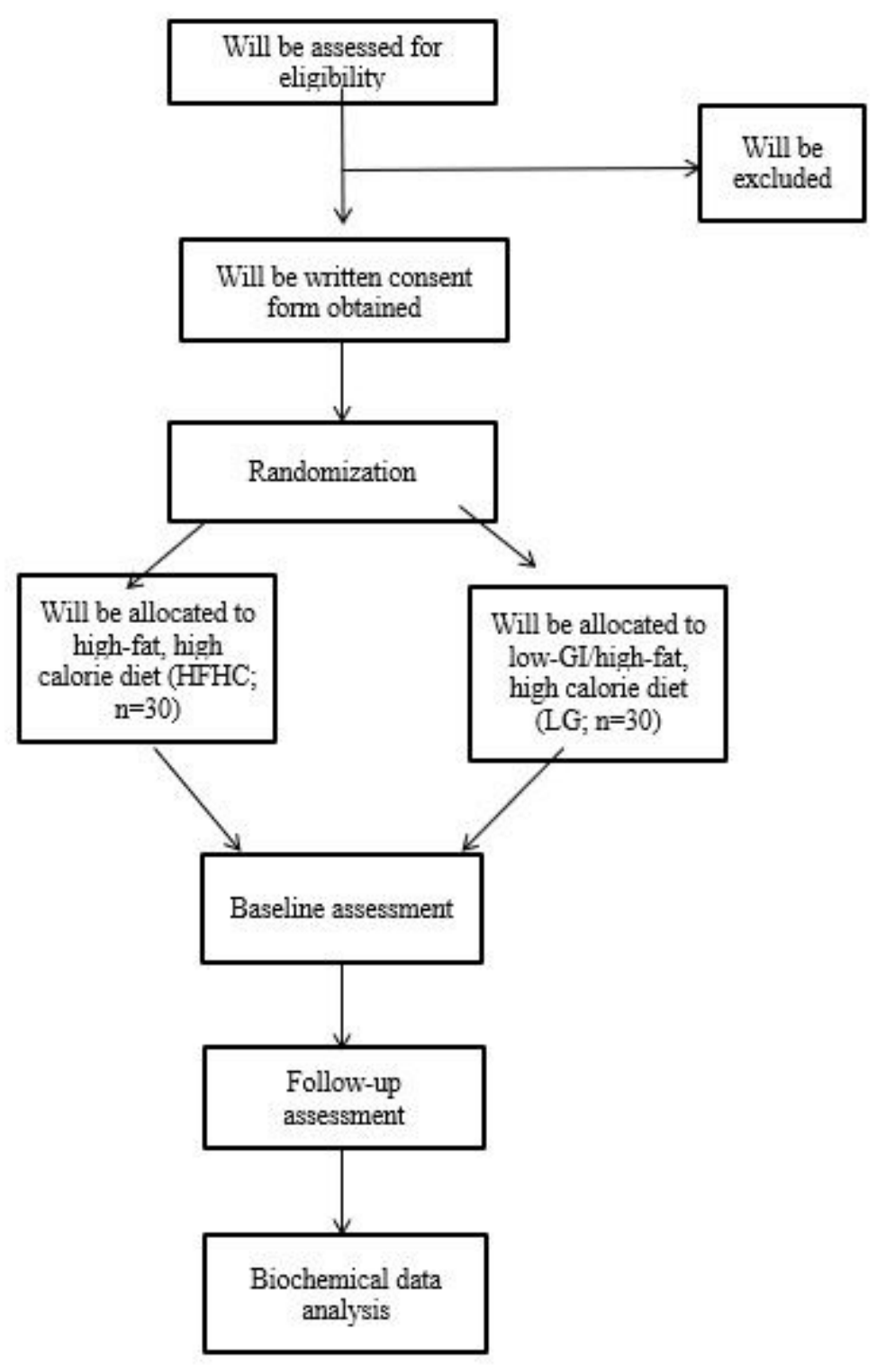

Figure 1. Study's flow diagram

Figure 1 


\begin{tabular}{|c|c|c|c|c|c|c|c|c|}
\hline \multirow[b]{3}{*}{ TIMEPOINT** } & \multicolumn{8}{|c|}{ STUDY PERIOD } \\
\hline & \multirow{2}{*}{$\frac{\text { Enrolment }}{-t_{1}}$} & \multirow{2}{*}{$\frac{\text { Allocation }}{0}$} & \multicolumn{5}{|c|}{ Post-allocation } & \multirow{2}{*}{$\frac{\text { Close-out }}{t}$} \\
\hline & & & $t_{1}$ & $t_{2}$ & $t_{3}$ & $t_{4}$ & etc. & \\
\hline \multicolumn{9}{|l|}{ ENROLMENT: } \\
\hline Eligibility screen & $\mathrm{X}$ & & & & & & & \\
\hline \multirow{2}{*}{$\begin{array}{l}\text { Informed consent } \\
\text { Demographic } \\
\text { measurements }\end{array}$} & $\mathrm{X}$ & & & & & & & \\
\hline & $\mathrm{X}$ & & & & & & & \\
\hline \multirow{2}{*}{$\begin{array}{l}\text { Anthropometric } \\
\text { measurements } \\
\text { Randomization }\end{array}$} & $\mathrm{X}$ & & & & & & & \\
\hline & & $\mathrm{x}$ & & & & & & \\
\hline Allocation & & $\mathrm{X}$ & & & & & & \\
\hline \multicolumn{9}{|l|}{ INTERVENTIONS: } \\
\hline \multicolumn{9}{|l|}{ [Intervention A] } \\
\hline \multicolumn{9}{|l|}{ [intervention B] } \\
\hline \multicolumn{9}{|l|}{ ASSESSMENTS: } \\
\hline $\begin{array}{l}\text { Rist baseline } \\
\text { variables] }\end{array}$ & $\mathrm{x}$ & $\mathrm{x}$ & & & & & & \\
\hline $\begin{array}{l}\text { [List outcome } \\
\text { variables] }\end{array}$ & & & $\mathrm{x}$ & $x$ & $\mathrm{x}$ & $\mathrm{x}$ & etc. & $\mathrm{X}$ \\
\hline $\begin{array}{l}\text { [List other data } \\
\text { variables] }\end{array}$ & & & $x$ & $\mathrm{X}$ & $\mathrm{X}$ & $x$ & etc. & $x$ \\
\hline
\end{tabular}

Figure 2. Time schedule of enrolment, interventions, assessments, and visits for participants.

\section{Figure 2}

Time schedule of enrolment, interventions, assessments, and visits for participants.

\section{Supplementary Files}

This is a list of supplementary files associated with this preprint. Click to download.

- SPIRITchecklist.doc 